\author{
Miguel Angel Silva \\ CRODE Celaya, Unidad de Metrología, México \\ miconsilva@yahoo.com.mx
}

\title{
Verificación del desempeño de una Máquina de Medición por Coordenadas utilizando el método de regla de pasos e interferometría
}

\section{Resumen}

En el presente trabajo, se reporta la obtención de los errores de indicación, obtenidos al realizar la verificación del desempeño de la Maquina de Medición por Coordenadas en mediciones lineales utilizando el método de regla de pasos medidos de manera bidireccional e interferometría unidireccional. En ambos casos, se aplico la norma ISO 10360-2:2009. Tanto el método de regla de pasos como el de interferometría, presentan consistencia en los resultados; en el primer método, el valor de la desviación es $30 \%$ mayor a los determinados por el segundo método. A partir de los análisis realizados, se determino que la calidad de la medición utilizando interferometria es mejor que el método de la regla de pasos. Esto es observable cuando se comparan los valores de incertidumbre arrojados de cada uno de los métodos. Siendo menor la estimada por interferometria.

Palabras Clave: Regla de pasos, Interferometría, Prueba de verificación en MMC, Norma ISO 103602:2009.

\begin{abstract}
In this work, indication errors are obtained through experimental verification of linear measurements about efficiency of the coordinate-measuring machine (CMM). Its measurements are carry out employing the method of step gage measured bidirectionally and unidirectional interferometry. The norm ISO 10360-2:2009 is used in both cases. Both the step gage method and interferometry, the results are according to data reported. In the first, step gage method, the deviation value is $30 \%$ greater than those determined by the second method. From the realizedanalyzes, it was determined that the measurements quality employing the interferometry method is better than step gage method. It can be observed when uncertainty values of each of the methods are compared, the uncertainty estimated through interferometry is less than the uncertainty estimated by the step gage method.
\end{abstract}

Key Words: Step gage, Interferometry, Verification test MMC, ISO 10360-2: 2009.

\section{Introducción.}

En la actualidad, el propósito básico de las mediciones dimensionales en la industria manufacturera (Schmid y Lazos Martinez, 2000; CENAM, 2016), es verificar y asegurar que el producto elaborado coincida con las especificaciones indicadas en el diseño (ISO 14253-1:2013), siendo la metrología, uno de los conductos para lograr este objetivo (LFMN, 2015). Por su 
versatilidad, las $\mathrm{MMC}^{\prime}$ s son utilizadas para la medición de piezas con geometrías complejas. En la actualidad, el avance tecnológico ha hecho que los diseños sean cada vez mas competitivos para optimizar los procesos, esto ha provocado que las mediciones para evaluar la conformidad respecto a tolerancias se dificulte. Por ello, las empresas adquieren MMC's para complementar su sistema metrológico. Como es sabido, todo instrumento de medición presenta errores de fabricación, que afectan la medición, a través de la verificación se cuantifica el error y la incertidumbre de la medición (NMX-CH-140-IMNC-2002); con el error, se realiza la compensación en la medición considerando la incertidumbre (NMX-Z-055-IMNC-2006). Así, se evita que productos fuera de especificación sean evaluados dentro de conformidad o viceversa. El procedimiento utilizado en la verificación del desempeño de una Máquina de Medición por Coordenadas (MMC), se describe a continuación: Primero, se eligen cinco distancias del patrón (instrumento de medición: regla de pasos o interferómetro), distribuidas a lo largo del alcance del patrón de longitud. Luego, realizar la compensación térmica en caso de aplicar; si es el caso, colocar el sensor térmico de la pieza de acuerdo a las recomendaciones de uso del fabricante. En seguida, se deja estabilizar térmicamente por un periodo mínimo de 2 horas. Registrar la temperatura ambiental dentro del volumen de medición de la CMM. A continuación, se caracteriza el sistema de palpado usando la esfera de referencia del fabricante, tratando que el desplazamiento de la punta del palpador del brazo soporte sea cero, o lo menor posible, para medir la longitud calibrada de la prueba. Posteriormente, se toma la temperatura del patrón durante la prueba y proceder a medir las cinco distancias definidas tres veces cada una. Repetir el punto anterior hasta completar las siete posiciones indicadas (ver tabla 1). Finalmente, se calcula el error de medición de longitud $\mathrm{E}_{0}$, calculando la diferencia entre la longitud medida y la calibrada para cada longitud de ensayo. Este procedimiento establece las pruebas para verificar el desempeño de CMMs usada para la medición dimensional como es establecido por el fabricante y de conformidad a las normas ISO 10360-2, ISO 10360-5 y ASME B89.4.10360-2 vigentes.

Tabla 1 - Especificaciones del volumen de medición.

\begin{tabular}{|c|c|c|}
\hline $\begin{array}{c}\text { Posición } \\
\text { número }\end{array}$ & Orientación en el volumen de medición & $\begin{array}{c}\text { Requerida o } \\
\text { recomendad } \\
\text { a }\end{array}$ \\
\hline 1 & Sobre la diagonal espacial desde $(1,0,0)$ al $(0,1,1)$ & Requerida \\
\hline 2 & Sobre la diagonal espacial desde $(1,1,0)$ al $(0,0,1)$ & Requerida \\
\hline 3 & Sobre la diagonal espacial desde $(0,1,0)$ al $(1,0,1)$ & Requerida \\
\hline 4 & Sobre la diagonal espacial desde $(0,0,0)$ al $(1,1,1)$ & Requerida \\
\hline 5 & Paralela a las escalas de la máquina desde $(0,1 / 2,1 / 2)$ al \\
$(1,1 / 2,1 / 2)$ & $\begin{array}{c}\text { Recomendad } \\
\text { a }\end{array}$ \\
\hline 6 & Paralela a las escalas de la máquina desde $(1 / 2,0,1 / 2)$ al \\
$(1 / 2,1,1 / 2)$ & $\begin{array}{c}\text { Recomendad } \\
\text { a }\end{array}$ \\
\hline 7 & Paralela a las escalas de la máquina desde $(1 / 2,1 / 2,0)$ al \\
$(1 / 2,1 / 2,1)$ & $\begin{array}{c}\text { Recomendad } \\
\text { a }\end{array}$ \\
\hline $\begin{array}{c}\text { NOTA: En esta tabla, las esquinas opuestas se asumen: }(0,0,0) \text { y }(1,1,1) \text { en coordenadas } \\
(X, Y, Z)\end{array}$ & \\
\hline
\end{tabular}

\section{Métodos de calibración}

\subsection{Patron regla de pasos medidos en una forma bidireccional.}

El error de indicación es la diferencia entre la longitud medida y la longitud calibrada, en este método la longitud de prueba calibrada se obtiene de la regla de pasos de la cual se conoce las dimensiones de prueba afectada por las condiciones ambientales. (fig. 1). 


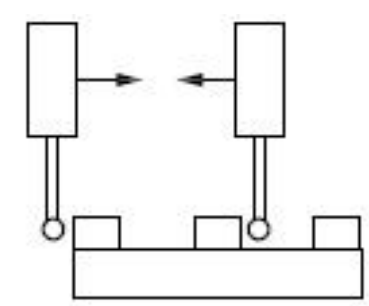

Figura 1 - Regla de pasos medición bidireccional.

\subsection{Interferometría laser medidos en forma unidireccional}

El error de indicación es la diferencia entre la longitud medida y la longitud calibrada, en este método la longitud de prueba calibrada es el desplazamiento de la MMC registrado por un sistema de interferómetro laser calibrado (fig. 2).

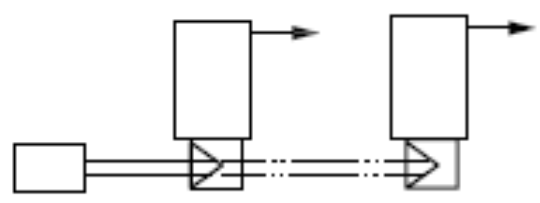

Figura 2 - Equipo de interfeomería medición unidireccional.

\section{Procedimiento de calibración de MMC.}

El principio del método de evaluación es utilizar una longitud de prueba calibrada, trazable al patrón Nacional de Longitud (el metro), que establezca si la MMC es capaz de medir dentro del error máximo permisible de longitud de medida especificado para una MMC dentro del límite máximo permisible indicado para el rango de repetibilidad. La evaluación debe ser llevada a cabo por comparación de los valores indicados de cinco diferentes longitudes de prueba calibradas, cada una medidas tres veces, relativas a sus valores calibrados. Los valores indicados son calculados por medidas de longitud de punto a punto proyectadas sobre la dirección de alineamiento.

\subsection{Procedimientos y posiciones de medición}

Se deben colocar cinco diferentes longitudes de prueba calibradas en cada una de siete diferentes posiciones (localizaciones y orientaciones) en el volumen de medición de MMC, y cada longitud debe ser medidas tres veces, para un total de 105 mediciones (ISO 10360-2:2009). Cuatro de las siete posiciones deben ser las diagonales en el espacio, como se muestra en figura. El usuario puede especificar el resto de las tres posiciones; las posiciones por entendido son paralelas a cada uno de los ejes de la MMC (fig. 3). 


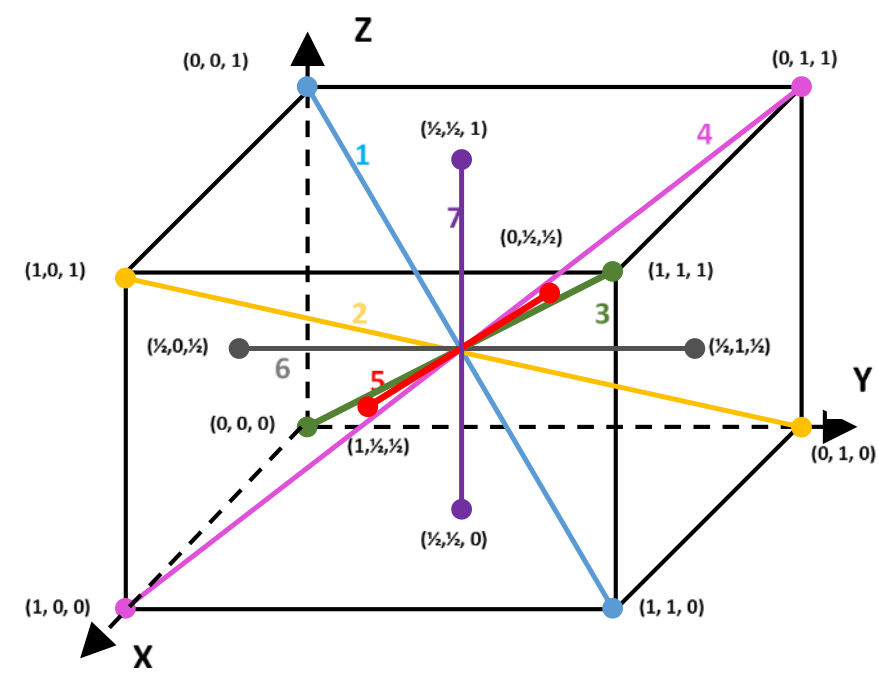

Figura 3 - Posiciones de medición

Cada una de las tres mediciones repetidas debe ser arreglada de la siguiente manera: $\mathrm{Si}$ un extremo de la longitud de prueba calibrada está marcado "A" y el otro extremo "B", entonces la secuencia de medición ya sea A1B1, A2B2, A3B3 ó A1B1, B2 A2, A3 B3. Otras secuencias como A1A2A3, B1B2B3 no están permitidos. Cada una de las tres mediciones repetidas tendrá sus únicos puntos de medida, i.e., en general, B1, B2 y B3 será diferentes puntos reales del mismo punto de destino B. Una vez iniciada la secuencia de medición del ensayo, ningún punto palpado adicional deberá ser medido, i.e., no se medirán puntos distintos de los necesarios para medir su longitud; e.g., no se permiten puntos de alineación entre la medición de A1 y B3 (ISO 10360-2:2009).

En una $\mathrm{MMC}$, si la longitud del eje más largo es al menos tres veces la longitud del eje intermedio, es recomendable incluir dos diagonales adicionales, cada una consistiendo de 5 longitudes, medida tres veces, en un plano perpendicular al eje más largo, i.e., si X es el eje más largo, entonces las dos diagonales están en el plano Y-Z y localizadas aproximadamente en el punto central del eje X. Más no son imprescindibles medirlas.

\section{Medición}

La medición es un conjunto de operaciones que tiene por objeto determinar el valor de una magnitud, en este caso, se require determinar el error de indicación (mensurando). A continuación se describe el procedimiento especifico: Primero, se colocar la regla de pasos y/o laser en la posición seleccionada. Alineando y/o ajustando, tanto la regla de pasos como el haz de luz respecto al recorrido de cada eje deseado o de cada línea de medición. Esta operación se realiza para evitar el error por sujeción. Véase ejemplo para el eje Y en la figura 4 (ISO 10360-2:2009). Ahora, para la regla de pasos se eligen 5 distancias para medir en la prueba, distribuidas a lo largo del patron de longitud, cuidando hacer al menos tres repeticiones de cada distancia. Para el Sistema interferometrico, seleccionar las longitudes de prueba, para cada unade las líneas de medición, inicializando las coordenadas a "Cero" en el software demedición. Estando la MMC en esa posición, inicializar el interferómetro láser en "Cero", de tal modo que los valores en ambos estén en "Ceros" (MMC en el eje a medir y el láser). Comprobar que la MMC en el eje a medir y el equipo laser, tengan el mismo sentido de conteo (+ó -). Cuando la MMC se mueva en un sentido, las coordenadas del láser deben marcar hacia el mismo sentido que la MMC. De estar invertidos los signos, cambiar en el láser el signo y repetir los pasos anteriores, de estar los signos igual continuar con el siguiente paso. Finalmente, realizar la medición. 


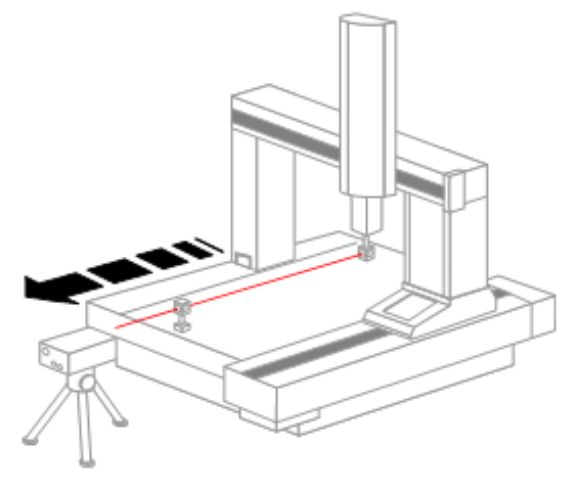

Figura 4 - Alineación para el eje Y.

\section{Cálculo del error de indicación}

La prueba debe ser llevada a cabo usando una longitud de compensación cero o tan pequeña como sea posible. Se reconoce que dependiendo del tipo de MMC, del artefacto de prueba y de la línea bajo evaluación L puede ser distinta de cero, pero esta deberá ser seleccionada la menor posible. La calibración se realizó en una máquina de medición de coordenadas con volumen efectivo de medición 1000x2100 x 600 mm³, Modelo Contura G2, Fabricante Carl Zeiss, Tipo Puente. Además, la regla de pasos utilizada tiene uan incertidumbre de medición de $(0.1+0.000004 \mathrm{~L}) \mathrm{L}$ en $\mathrm{mm}$, material Acero con alcance de medición de $700 \mathrm{~mm}$ (ver fig. 5). El interferómetro es de Helio-Neón por efecto Zeeman de doble frecuencia, cuenta con una exactitud $\pm 0.4 \mathrm{ppm}$ con un alcance de medición 80 metros. La temperatura promedio durante la prueba fue de $20.6{ }^{\circ} \mathrm{C}$ con una humedad relativa promedio de $46 \%$.

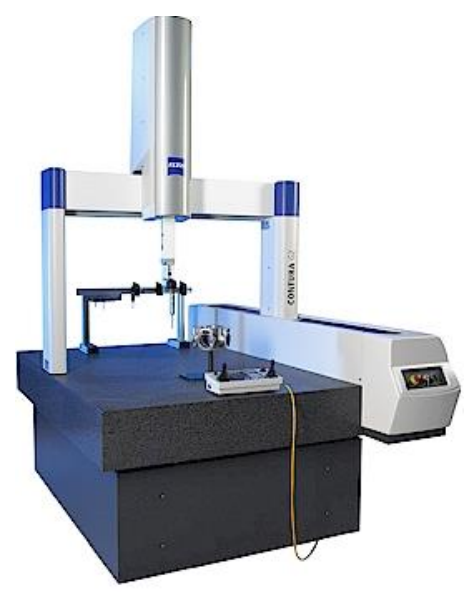

Figura 5 - Máquina de medición de coordenadas. (Cortesía de CAPYMET, S.A de C.V.).

\section{Resultados}

Regla de pasos. Para obtener las longitudes calibradas, se aplico el procedimiento de compensación de los errores de temperatura y calibración. Posteriormente, se realizaron las tres mediciones reglamentarias de acuerdo a la norma. Luego, se determino la desviación en cada una de las mediciones, obteniendo la diferencia entre cada una de las longitudes medidas y la longitude calibrada. El valor de la desviación es comparado con el error máximo permitido de ma MMC, asignado por el fabricante. Por útimo se determina la incertidumbre de medida en cada longitud calibrada con un nivel de confianza del $95,45 \%$. A continuación se presentan, en la tabla 2 , los resultados obtenidos. 
Tabla 2 - Error de indicación en el eje "Y" utilizando regla de pasos.

\begin{tabular}{|c|c|c|c|c|c|c|c|c|}
\hline \multirow{2}{*}{$\begin{array}{l}\text { Longitudes } \\
\text { calibradas }\end{array}$} & \multicolumn{3}{|c|}{ Longitudes medidas } & \multirow[t]{2}{*}{$U$} & \multirow{2}{*}{$\begin{array}{c}\mathrm{E} 0, \mathrm{MPE}= \\
\pm(1.9+\mathrm{L} / 300) \\
\text { Len } \mathrm{mm}\end{array}$} & \multicolumn{3}{|c|}{ Desviación medida } \\
\hline & 1 & 2 & 3 & & & 1 & 2 & 3 \\
\hline$(\mathrm{mm})$ & $(\mathrm{mm})$ & $(\mathrm{mm})$ & $(\mathrm{mm})$ & $(\mu \mathrm{m})$ & $(\mu \mathrm{m})$ & $(\mu \mathrm{m})$ & $(\mu \mathrm{m})$ & $(\mu \mathrm{m})$ \\
\hline 1948.6248 & 1948.62616 & 1948.62642 & 1948.62626 & 3.25 & 8.4 & 1.36 & 1.62 & 1.46 \\
\hline 1598.6708 & 1598.67400 & 1598.67399 & 1598.67402 & 2.73 & 7.23 & 3.20 & 3.19 & 3.22 \\
\hline 1198.7096 & 1198.71278 & 1198.71282 & 1198.71281 & 2.13 & 5.9 & 3.18 & 3.22 & 3.21 \\
\hline 749.8860 & 749.88677 & 749.88681 & 749.88695 & 1.45 & 4.4 & 0.77 & 0.81 & 0.95 \\
\hline 349.9855 & 349.98350 & 349.98347 & 349.98347 & 0.85 & 3.07 & -2.00 & -2.03 & -2.03 \\
\hline
\end{tabular}

Interferometría. La metodología utilizada en la obtención de estos resultados, es equivalente a la utilizada en el caso de Regla de pasos. A continuación, se presentan los resultados (tabla 3).

Tabla 3 - Error de indicación en el eje "Y" utilizando interferometría.

\begin{tabular}{|c|c|c|c|c|c|c|c|c|}
\hline \multirow{2}{*}{$\begin{array}{l}\text { Longitudes } \\
\text { calibradas }\end{array}$} & \multicolumn{3}{|c|}{ Longitudes medidas } & \multirow[t]{2}{*}{ U } & \multirow{2}{*}{$\begin{array}{c}E 0, M P E= \\
\pm(1.9+L / 300) \\
\text { Len } \mathrm{mm}\end{array}$} & \multicolumn{3}{|c|}{ Desviación medida } \\
\hline & 1 & 2 & 3 & & & 1 & 2 & 3 \\
\hline$(\mathrm{mm})$ & $(\mathrm{mm})$ & $(\mathrm{mm})$ & $(\mathrm{mm})$ & $(\mu \mathrm{m})$ & $(\mu \mathrm{m})$ & $(\mu \mathrm{m})$ & $(\mu \mathrm{m})$ & $(\mu \mathrm{m})$ \\
\hline 1948.62868 & 1948.62877 & 1948.62879 & 1948.62873 & 0.97 & 8.4 & 0.09 & 0.11 & 0.05 \\
\hline 1598.6923 & 1598.6914 & 1598.69129 & 1598.69135 & 0.80 & 7.23 & -0.90 & -1.01 & -0.95 \\
\hline 1198.7221 & 1198.72233 & 1198.72225 & 1198.72219 & 0.60 & 5.9 & 0.23 & 0.15 & 0.09 \\
\hline 749.9220 & 749.92089 & 749.92094 & 749.92098 & 0.37 & 4.4 & -1.11 & -1.06 & -1.02 \\
\hline 349.99283 & 349.99216 & 349.99252 & 349.99202 & 0.17 & 3.07 & -0.67 & -0.31 & -0.81 \\
\hline
\end{tabular}

La representación grafica de los errores de indicación y su incertidumbre asociada, indican la conformidad de las desviaciones en todas las longitudes medidas, respecto al error máximo tolerado permisible. El error por interferometría es mas exacto y su incertidumbre de medición es menor.

(a)

(b)

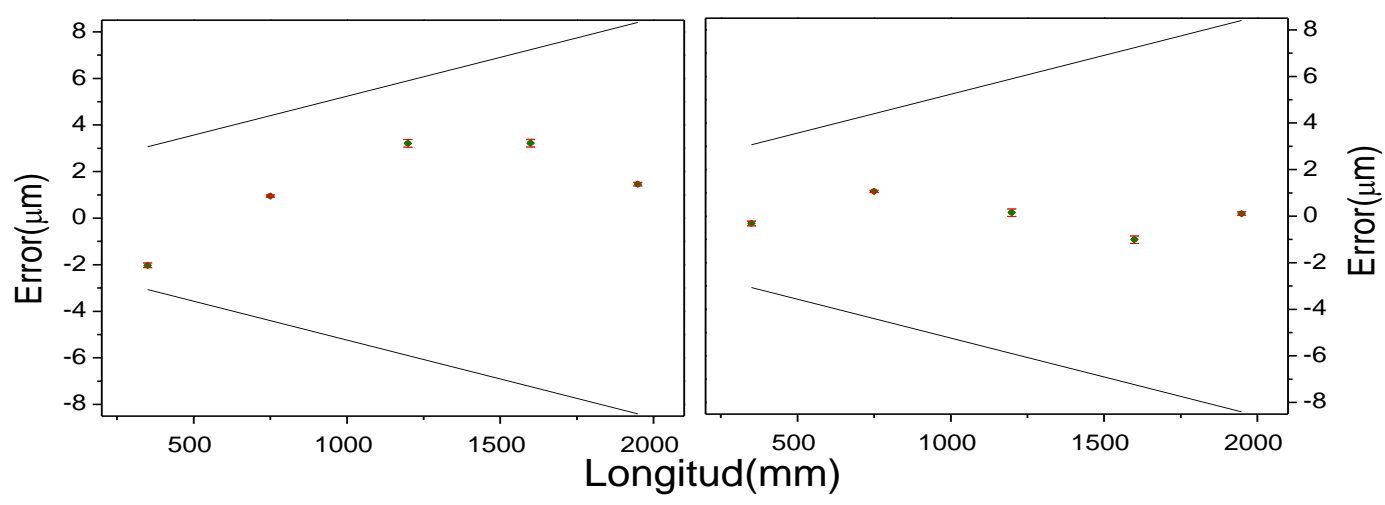

Figura 5 - Error evaluado en cada posición. (a) con regla de pasos y (b) interferometría.

\section{Conclusiones}

A partir del análisis realizado, se determino que la Máquina de Medición por Coordenadas se encuentra dentro de los errores máximos tolerados, especificados por el fabricante. Tanto el método de regla de pasos como el de interferometría, presentan consistencia en los resultados; en el caso de la regla de pasos, el valor de la desviación es 30\% mayor a los encontrados por el método de interferometria. De la misma forma, la calidad de la medición utilizando interferometria es mejor que el método de la regla de pasos. Esto es observable cuando se comparan los valores de incertidumbre 
arrojados de cada uno de los métodos. Siendo menor la estimada por interferometria. Por otra parte, es importante mencionar que ambos métodos son confiables, sin embargo, es claro que a mayor calidad de medición, mayor deberá ser el costo de los instrumentos debido a su exactitud de medida; siendo mas práctico realizar la verificación a Maquinas de Medición por Coordenadas de alto alcance con el método interferometrico.

\section{Agradecimientos}

Los autores desean agradecer al Fondo de Innovación del CONCYTEG por el apoyo brindado para la realización de este proyecto convenio 0045.

\section{Referencias}

DE METROLOGíA, Centro Español. Vocabulario Internacional de Metrología Conceptos fundamentales y generales, y términos asociados (VIM). 2012.

CENAM. Metrología

Dimensional. Disponível

em: <http://www.cenam.mx/dimensional/dimensional.aspx>. Acesso em: 05 mar. 2015.

W. A. Schmid y R. J. Lazos Martinez. Guía técnica para estimar la incertidumbre de medición. CENAM, 2000.

LFMN, Ley Federal sobre Metrología y Normalización, 2015.

ICONTEC, GTC. Guía para la expresión de incertidumbre en las mediciones. 2003. E: Guide to the expression of uncertainty in measurement, 51.

ISO 14253. Geometrical Product Specifications (GPS) - Inspection by measurement of workpieces and measuring equipment: Part 1: Decision rules for proving conformance or nonconformance with specifications, 2013.

SPECIFICATIONS, Geometrical Product. Acceptance and Reverification Tests for Coordinate Measuring Machines (CMM) - Part 2: CMMs Used for Measuring Linear Dimensions, ; ISO 10360-2: 2009. International Organazation for Standardization: Geneva, Switzerland, 2009.

NMX-CH-14253-2-IMNC-2005 Especificaciones Geométricas de Producto (GPS) -Inspección por medición de piezas de trabajo y de equipo de medición - Parte 2: Guía para la estimación de la incertidumbre en medición de GPS, en la calibración de equipo de medición.

SOBRE TRAZABILIDAD, Guia Tecnica. incertidumbre en Metrologia Dimensional. EMA, Revision, v. 1. 